\title{
A Linearized Analytical Solution Of PDE Heat Transfer In A Solid Material Excited By Light With Radiant Thermal Losses
}

DOI: $10.46932 / \mathrm{sjjdv3n1-035}$

Received in: Dec 30st, 2021

Accepted in: Jan 1th, 2022

\section{Raúl González-García \\ Doctorado en Ciencias en Ingeniería Química}

Faculty of Chemical Sciences, Autonomous University of San Luis Potosi Av. Dr. Manuel Nava No. 6, Zona Universitaria, San Luis Potosí 78210, México

E-mail: raulgg@uaslp.mx

Rumen I. Tsonchev

Doctorado en Ciencias Técnicas

Academic Unit of Physics, Autonomous University of Zacatecas

Calz. Solidaridad, Esquina Paseo de la Bufa s/n, Zacatecas 98060, Mexico

E-mail: rumen5252@yahoo.com.mx

\section{Miguel A. Ruiz-Cabrera}

Doctorado en Ciencia de Alimentos

Faculty of Chemical Sciences, Autonomous University of San Luis Potosi

Av. Dr. Manuel Nava No. 6, Zona Universitaria, San Luis Potosí 78210, México

E-mail: mruiz@uaslp.mx

\section{Alicia Grajales-Lagunes}

Doctorado en Ciencia de Alimentos

Faculty of Chemical Sciences, Autonomous University of San Luis Potosi

Av. Dr. Manuel Nava No. 6, Zona Universitaria, San Luis Potosí 78210, México

E-mail: grajales@uaslp.mx

José J. Villa-Hernández

Doctorado en Ciencias (Óptica)

Academic Unit of Electrical Engineering, Autonomous University of Zacatecas Av. Ramón López Velarde 801, Zacatecas 98000, Mexico

E-mail: jvillah@yahoo.com

\section{Daniel Alaniz-Lumbreras}

Doctorado en Ingeniería

Academic Unit of Electrical Engineering, Autonomous University of Zacatecas Av. Ramón López Velarde 801, Zacatecas 98000, Mexico

E-mail:dalaniz@uaz.edu.mx

\begin{abstract}
A method to linearize partial differential equations (PDEs) of heat diffusion and its initial with boundary conditions in a wide interval of temperature is presented. Thus, an analytical solution of the heat conduction problem was achieved in a virtual experiment performed in a lead wall with an ideally established configuration. For other arrangements where an analytical solution is not possible, simple
\end{abstract}


standard programs can be used as an alternative to solve the linearized partial differential equations obtained with the proposed method. A sophisticated computer program as the standard gold method of COMSOL Multiphysics ${ }^{\circledR}$ was used for the evaluation of error of procedure. It was validated that the relative error of the analytical solution, in comparison to the digital simulation with COMSOL Multiphysics, can be found without the need to own and use the COMSOL Multiphysics program. For our configuration, the error is $1.16 \%$ and focuses on the initial part of the process.

Keywords: Heat transfer, PDE, Radiant losses, Analytical solution.

\section{INTRODUCTION}

In order to find the thermal properties of a material, it is mandatory to conduct thermal experiments whose theoretical foundation are the partial differential equations (PDEs) of heat transfer [1]. In many cases, when working on a wide interval of temperature, these PDEs are nonlinear because the thermal properties of the materials and the heat transfer coefficient $h\left[\mathrm{Wm}^{-2} \mathrm{~K}^{-1}\right]$ are dependent on the temperature. Besides, the heat loss coefficient involves the sum of three components, as shown in Eq. 1:

$$
h=h_{\text {conv }}+h_{\text {cond }}+h_{\text {rad }}
$$

where $h_{\text {conv }}, h_{\text {cond }}$ and $h_{\text {rad }}$ represent heat exchange with the environment: the heat loss due to thermal convection, thermal conduction, and thermal radiation, respectively. It is known that $h_{\text {conv }}$ and $h_{\text {cond }}$ components are significative affected by the conditions of the experiment such as the construction of the measurement arrangement [2-4]; the air velocity in the measurement laboratory [5]; the type of air movement - laminar or turbulent [6]; the emissivity of the sample surface [7]; the roughness of the sample [8-9], the local temperature of the sample [10-11], etc. Such conditions make the experimental measurement, prediction, and theoretical description of both components be very hard and even impractical. However, $h_{\text {rad }}$ part can be expressed with the Stefan-Boltzmann Law [12] and using short calculations the Eq. 2 can be obtained:

$$
h_{\text {rad,exact }}=\varepsilon \sigma\left(T^{2}+T_{0}^{2}\right)\left(T+T_{0}\right)
$$

where $T[\mathrm{~K}]$ is the experimental temperature, $T_{0}[\mathrm{~K}]$ is the environment temperature, $\sigma$ is the Stefan-Boltzmann constant, $\sigma=5.670373 \times 10^{-8}\left[\mathrm{Wm}^{-2} \mathrm{~K}^{-4}\right], \varepsilon$ represents the thermal emissivity of the sample. For accurate and reliable results, it is preferable to work under a vacuum where it can be assumed that $h_{\text {conv }}=h_{\text {cond }}=0$ [13-16]. Such parameters can be considered constant when the temperature 
difference, $T-T_{0}$, is small compared to the ambient temperature, $T_{0}$ [17-19]. However, $h_{\text {rad }}$ can be approximated by expansion in the Taylor series, with the Eq. 3:

$$
h_{\text {rad,approx }}=4 \varepsilon \sigma T_{0}^{3}
$$

To be able to use the approximation indicated by Eq. 3, it is necessary to work in a narrow interval of temperatures around the environment temperature. However, it has been noticed that when the experimental temperature $T$ significative increases over $T_{0}$ the errors provoked by the use of these approximations rapidly become unacceptable. That is shown in Fig. 1 where the relative error calculated with Eq. 4 as a function of the relative change of temperature $\left(T-T_{0}\right) / T_{0}$ was plotted:

$$
\text { Relative error }[\%]=\frac{h_{\text {rad,exact }}-h_{\text {rad,aprox }}}{h_{\text {rad,exact }}} \times 100
$$

From this perspective, in case of a big difference, $T-T_{0}$, the use of the approximation given by Eq. 3 is not permitted.

Sophisticated programs such as the program COMSOL Mutiphysics ${ }^{\circledR}$ are generally used as the gold method to resolve in a precise way the nonlinear PDEs [20]. Therefore, in this paper is presented a simplified approach to linearize the PDE mainly related to heat diffusion. The objective of this paper is to find an analytical solution to the heat conduction problem in order to achieve similar results to those obtained with the standard gold method of COMSOL Mutiphysics@, which uses nonlinear PDEs. At the same time, the variation of the thermal parameters of the material and heat loss coefficient with the temperature will be taken into account. A virtual experiment will be done to prove the usefulness of the proposed method as well as to determine the discrepancy values with the exact solution.

Figure 1. Relative error as a function of the relative change of temperature

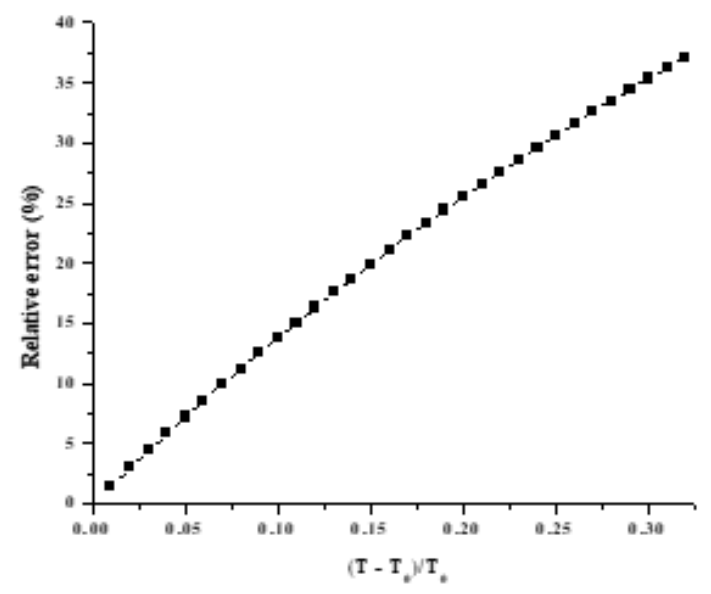




\section{DESCRIPTION OF THE VIRTUAL EXPERIMENT}

The virtual experimental arrangement consists of a lead wall with two infinite dimensions and with finite, but thermally immense, thickness, with flat interfaces in the vacuum, as schematically presented in Fig. 2.

Figure 2. Structure of the virtual experimental arrangement

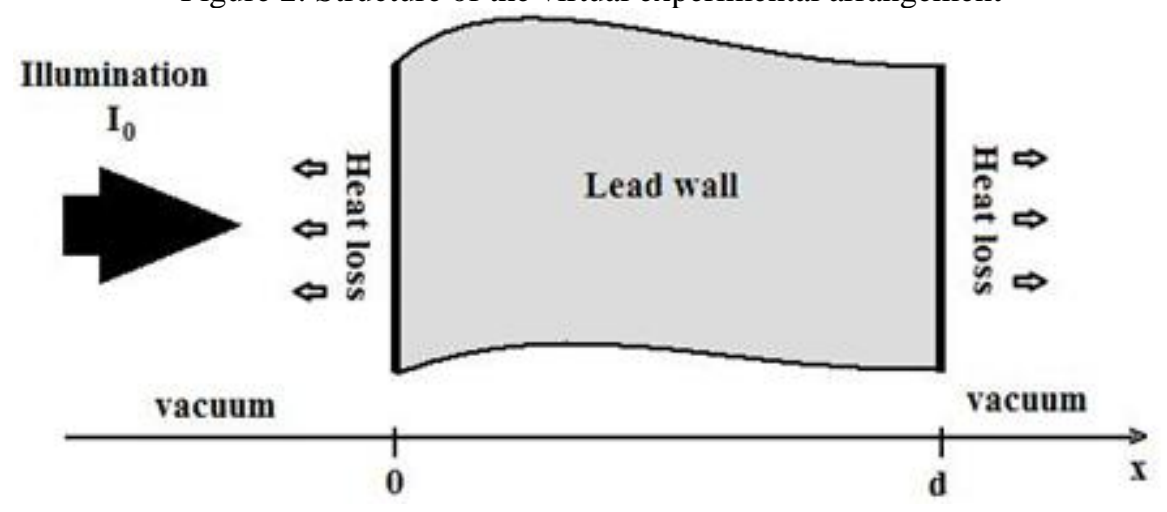

Concerning Fig. 2, it was assumed that (a) heat transfer takes place in one direction in regards to the thickness. (b) There was only radiation energy loss in both walls $(x=0$ and $x=d)$. (c) The chosen thickness of the wall was so big, $d=10 \mathrm{~m}$, to guarantee that in the rear face, heat losses were relatively minor. Thus, for $x=d$, a heat loss coefficient value of approximately zero was considered. (d) Both interfaces were painted with optically diffused black paint (model 359 black, COMEX) [21], to ensure a thermal emissivity $\varepsilon=0.96$ [22]. (e) The interface in $x=0$ was heated by a uniform and constant light after its start, with intensity $I_{0}\left[\mathrm{~W} / \mathrm{m}^{2}\right]$, coming from the left zone of the vacuum. (f) At the moment $t=$ 0 , the light was turned on, before $t=0$, the light was deliberately off. (g) The experimental temperature interval chosen for the left interface of the lead wall was relatively wide: minimum temperature $T_{1}=300$ $\mathrm{K}$, maximum temperature $T_{2}=600 \mathrm{~K}$. A maximum simulation time of $6000 \mathrm{~s}$ was used for the virtual experiment. Evidently, in such cases, the approximation is given by Eq. 3 cannot be used.

Based on the assumptions above, the heat transfer in the lead wall was only governed by conduction phenomena described by Eq. 5:

$$
\rho C_{p} \frac{\partial T}{\partial t}=\nabla(k \nabla T)
$$

for unidimensional heat conduction, Eq. 5 was reduced to the following spatial-temporal PDE:

$$
\rho C_{p} \frac{\partial T}{\partial t}=\frac{\partial}{\partial x}\left(k \frac{\partial T}{\partial t}\right)
$$


subjected to the following nonlinear boundary and initial conditions:

$$
\begin{aligned}
& -k \frac{\partial T}{\partial x}=\sigma A \varepsilon\left(T_{0}^{4}-T^{4}\right)+q_{0} \quad \text { in } \quad x=0 \\
& -k \frac{\partial T}{\partial x}=\sigma A \varepsilon\left(T^{4}-T_{0}^{4}\right) \quad \text { in } \quad x=d \\
& T=T_{0} \quad \text { for } \quad t=0
\end{aligned}
$$

where $q_{0}$ represents the energy flux contributed by the incident light. From the above boundary conditions (Eq. 7 to 9), it can be appreciated that the effect of heat transfer by convection was not included because both sides of the wall were exposed to the vacuum. It can also be noticed that as a consequence of the nonlinearity of the boundary conditions and the dependence of the thermal parameters of the metal on the temperature, there is not a direct analytical solution of Eq. 6.

\section{THEORY TO FIND AN ANALYTICAL SOLUTION}

Among the main thermal properties of the lead wall to find the analytical solution was: $\rho$ - the mass density of the material $\left[\mathrm{kg} / \mathrm{m}^{3}\right], c_{p}$ - the specific heat capacity $[\mathrm{J} /(\mathrm{kg} \cdot \mathrm{K})], k$ - the thermal conductivity $[\mathrm{W} /(\mathrm{m} \cdot \mathrm{K})], h_{\text {rad }}-$ the radiation heat loss coefficient $\left[\mathrm{W} /\left(\mathrm{m}^{2} \cdot \mathrm{K}\right)\right]$, all of them as a function of temperature $[20]$.

To achieve an analytical solution of the heat diffusion PDE, it is necessary to obtain constant values of the thermal properties $\rho, c_{p}, k$ and $h_{\text {rad }}$. Therefore, it is proposed to calculate their statistical average values in the range of working temperatures, $T_{1}$ and $T_{2}$, using mathematical functions for $\rho(T)$, $c_{p}(T)$ y $k(T)$ from the literature [20] according to Eq. 10 to 13:

$$
\begin{aligned}
h_{\text {rad }}^{\text {stat }}= & \frac{1}{T_{2}-T_{1}} \int_{T_{1}}^{T_{2}} h_{\text {rad }}(T) d T \\
& =\frac{\varepsilon \sigma}{T_{2}-T_{1}}\left(\frac{1}{4} T^{4}+\frac{1}{3} T_{0} * T^{3}+\frac{1}{2} T_{0}^{2} * T^{2}+T_{0}^{3} * T\right)_{T_{1}}^{T_{2}} \\
\rho^{\text {stat }}= & \frac{1}{T_{2}-T_{1}} \int_{T_{1}}^{T_{2}} \rho(T) * d T \\
c_{p}^{\text {stat }}= & \frac{1}{T_{2}-T_{1}} \int_{T_{1}}^{T_{2}} c_{p}(T) * d T \\
k^{\text {stat }}= & \frac{1}{T_{2}-T_{1}} \int_{T_{1}}^{T_{2}} k(T) * d T
\end{aligned}
$$


Working temperatures, $T_{1}$ and $T_{2}$, will give, in the case of the absence of the program COMSOL Multiphysics, the limits of the analytical solution and will allow to evaluate the maximum error of the calculations.

The PDE for unidimensional heat conduction (Eq. 6), as well as the boundary and initial conditions, are expressed in their respective linearized form as:

$$
\begin{aligned}
& \frac{\partial T}{\partial t}=\frac{k}{\rho C_{p}} \frac{\partial^{2} T}{\partial x^{2}} \\
& -k \frac{\partial T}{\partial x}=h_{r a d}\left(T_{0}-T\right)+q_{0} \quad \text { in } x=0 \\
& -k \frac{\partial T}{\partial x}=h_{\text {rad }}\left(T-T_{0}\right) \quad \text { in } x=d \\
& T=T_{0} \quad \text { for } t=0
\end{aligned}
$$

Consequently, heat transfer in the lead wall was solved analytically through the non-trivial solution procedure that involved the following steps:

1.- Obtain dimensionless equations. With the following definitions:

$$
U=T-T_{0}, \quad X=\frac{x}{d}, \quad N_{B i}=\frac{h_{r a d^{*} d}}{k}, \quad \tau=\frac{t}{d^{2}}, \quad Q_{0}=\frac{q_{0}}{h_{\text {rad }}}, \quad \alpha=\frac{k}{\rho C_{p}}
$$

the tansformed version of the heat conduction problem becomes:

$$
\frac{\partial U}{\partial \tau}=\alpha \frac{\partial^{2} U}{\partial X^{2}}
$$

where:

$$
\begin{aligned}
& \frac{\partial U}{\partial X}=N_{B i}\left(U-Q_{0}\right) \text { in } X=0 \\
& \frac{\partial U}{\partial X}=-N_{B i} U \text { in } X=1 \\
& U=0 \quad \text { for } \quad \tau=0
\end{aligned}
$$

2.- Application of the principle of superposition. In this case, the following definition was used

$$
U=U_{s}(X)+\widehat{U}(\tau, X)
$$

where $U_{s}$ and $\widehat{U}$ are the solution in steady-state and the contribution of the transient state, respectively. The two following problems were obtained: 


\section{Problem 1}

$\frac{\partial^{2} U_{S}}{\partial X^{2}}=0$

Subject to

$$
\frac{\partial U_{S}}{\partial X}=N_{B i}\left(U_{s}-Q_{0}\right) \quad \text { in } \quad X=0
$$

$$
\frac{\partial U_{s}}{\partial X}=-N_{B i} U_{s} \text { in } X=1
$$

3.- Resolution of the problems 1 and 2 through the separation of variables:

The solution for the ordinary differential equation (ODE) of Problem 1 was:

$$
U_{s}(X)=C_{1} X+C_{2}
$$

and by applying its corresponding boundary conditions, it was obtained:

$$
U_{s}(X)=\frac{-Q_{0}}{2+N_{B i}}\left(N_{B i} X-1-N_{B i}\right)
$$

The solution for the PDE of Problem 2 was found as:

$$
\widehat{U}=\sum_{n=1}^{\infty} P_{n}\left(\cos \left(\lambda_{n} X\right)+\frac{N_{B i}}{\lambda_{n}} \sin \left(\lambda_{n} X\right)\right) e^{-\lambda_{n}^{2} \alpha \tau}
$$

whereas the variable $\lambda_{n}$ was obtained from the solution set of the characteristic equation:

$$
2 N_{B i}\left(\frac{\lambda}{\lambda^{2}-N_{B i}^{2}}\right)-\tan \lambda=0
$$

Finally, based on the information of the phenomenon, the solution can be obtained by applying the following algorithm:

$$
N_{B i}=\frac{\bullet}{{\stackrel{h}{r a d^{*} d}}} \text { Calculate the parameter: }
$$

- Get all characteristic values from: 


$$
2 N_{B i}\left(\frac{\lambda_{n}}{\lambda_{n}^{2}-N_{B i}^{2}}\right)-\tan \left(\lambda_{n}\right)=0 \quad \forall n=1,2,3, \cdots, \infty
$$

- $\quad$ Calculate the parameters:

$$
X=\frac{x}{d} ; \quad \tau=\frac{t}{d^{2}} ; \quad Q_{0}=\frac{q_{0}}{h_{\text {rad }}} ; \quad \alpha=\frac{k}{\rho C_{p}}
$$

- $\quad$ Calculate the steady solution:

$$
U_{S}(X)=\frac{Q_{0}}{2+N_{B i}}\left(1+N_{B i}-N_{B i} X\right)
$$

- Calculate the truncated transient solution in a sufficient number of terms, $n_{u}$ :

$$
\begin{aligned}
& P_{n}=\frac{-2 Q_{0}}{2+N_{B i}} \frac{\left(\lambda_{n}^{2}-N_{B i}^{2}\right) \sin \lambda_{n}+2 N_{B i} \lambda_{n}\left(1-\cos \lambda_{n}\right)+N_{B i}^{2} \lambda_{n}}{\left(\lambda_{n}^{2}-N_{B i}^{2}\right) \cos \lambda_{n} \sin \lambda_{n}+2 N_{B i} \lambda_{n}\left(1-\cos ^{2} \lambda_{n}\right)+N_{B i}^{2} \lambda_{n}+\lambda_{n}^{3}} \\
& \widehat{U}(\tau, X)=\sum_{n=1}^{n_{u}} P_{n}\left(\cos \left(\lambda_{n} X\right)+\frac{N_{B i}}{\lambda_{n}} \sin \left(\lambda_{n} X\right)\right) e^{-\lambda_{n}^{2} \alpha \tau}
\end{aligned}
$$

of Eq. 18:

$$
T(\tau, X)=T_{0}+U_{s}(X)+\widehat{U}(\tau, X)
$$

In the virtual experiment, the following parameters were used:

$$
\begin{array}{ll}
T_{0}=T_{1}=300 \mathrm{~K} & T_{2}=600 \mathrm{~K} \\
d=10 \mathrm{~m} & \rho=11218 \mathrm{~kg} / \mathrm{m}^{3} \\
C_{p}=136.42 \mathrm{~J} /(\mathrm{kg} \mathrm{K}) & k=33.3745 \mathrm{~W} /(\mathrm{m} \mathrm{K}) \\
h_{\text {rad }}=13.141 \mathrm{~W} /\left(\mathrm{m}^{2} \mathrm{~K}\right) & I_{0}=30000 \mathrm{~W} / \mathrm{m}^{2}
\end{array}
$$

It is crucial to notice that Eq. 10 to 13 with temperatures values of $T_{1}=T_{0}=300 \mathrm{~K}$ and $T_{2}=600$ $\mathrm{K}$ were applied to determine the values of the mass density, specific heat capacity, thermal conductivity, and of the radiation heat loss coefficient.

On the other hand, the following composed parameters were calculated:

$$
\begin{aligned}
& N_{B i}=h * d / k=3.937467 \\
& Q_{0}=q_{0} / h_{r a d}=2282.914 \mathrm{~K} \\
& \alpha=k /\left(\rho * C_{p}\right)=2.18 \mathrm{E}-05 \mathrm{~m}^{2} / \mathrm{s}
\end{aligned}
$$

and also the first 58 characteristic values, obtained from the solution of Eq. 27, were considered and are presented in the following table: 
Table 1. The first 58 characteristic values obtained with Eq. 37

\begin{tabular}{|l|l|l|l|r|r|}
\hline $\mathbf{n}$ & $\boldsymbol{\lambda}_{\boldsymbol{n}}$ & $\boldsymbol{n}$ & $\boldsymbol{\lambda}_{\boldsymbol{n}}$ & $\boldsymbol{n}$ & $\boldsymbol{\lambda}_{\boldsymbol{n}}$ \\
\hline $\mathbf{1}$ & 2.1443 & $\mathbf{2 1}$ & 62.9568 & $\mathbf{4 1}$ & 125.7263 \\
\hline $\mathbf{2}$ & 4.5650 & $\mathbf{2 2}$ & 66.0925 & $\mathbf{4 2}$ & 128.8664 \\
\hline $\mathbf{3}$ & 7.2753 & $\mathbf{2 3}$ & 69.2287 & $\mathbf{4 3}$ & 132.0065 \\
\hline $\mathbf{4}$ & 10.1640 & $\mathbf{2 4}$ & 72.3653 & $\mathbf{4 4}$ & 135.1467 \\
\hline $\mathbf{5}$ & 13.1483 & $\mathbf{2 5}$ & 75.5024 & $\mathbf{4 5}$ & 138.2870 \\
\hline $\mathbf{6}$ & 16.1852 & $\mathbf{2 6}$ & 78.6399 & $\mathbf{4 6}$ & 141.4273 \\
\hline $\mathbf{7}$ & 19.2530 & $\mathbf{2 7}$ & 81.7776 & $\mathbf{4 7}$ & 144.5677 \\
\hline $\mathbf{8}$ & 22.3401 & $\mathbf{2 8}$ & 84.9157 & $\mathbf{4 8}$ & 147.7082 \\
\hline $\mathbf{9}$ & 25.4399 & $\mathbf{2 9}$ & 88.0540 & $\mathbf{4 9}$ & 150.8486 \\
\hline $\mathbf{1 0}$ & 28.5484 & $\mathbf{3 0}$ & 91.1925 & $\mathbf{5 0}$ & 153.9892 \\
\hline $\mathbf{1 1}$ & 31.6634 & $\mathbf{3 1}$ & 94.3312 & $\mathbf{5 1}$ & 157.1297 \\
\hline $\mathbf{1 2}$ & 34.7830 & $\mathbf{3 2}$ & 97.4701 & $\mathbf{5 2}$ & 160.2704 \\
\hline $\mathbf{1 3}$ & 37.9061 & $\mathbf{3 3}$ & 100.6092 & $\mathbf{5 3}$ & 163.4110 \\
\hline $\mathbf{1 4}$ & 41.0320 & $\mathbf{3 4}$ & 103.7484 & $\mathbf{5 4}$ & 166.5517 \\
\hline $\mathbf{1 5}$ & 44.1602 & $\mathbf{3 5}$ & 106.8878 & $\mathbf{5 5}$ & 169.6924 \\
\hline $\mathbf{1 6}$ & 47.2900 & $\mathbf{3 6}$ & 110.0273 & $\mathbf{5 6}$ & 172.8332 \\
\hline $\mathbf{1 7}$ & 50.4213 & $\mathbf{3 7}$ & 113.1669 & $\mathbf{5 7}$ & 175.9739 \\
\hline $\mathbf{1 8}$ & 53.5539 & $\mathbf{3 8}$ & 116.3066 & $\mathbf{5 8}$ & 179.1147 \\
\hline $\mathbf{1 9}$ & 56.6874 & $\mathbf{3 9}$ & 119.4464 & & \\
\hline $\mathbf{2 0}$ & 59.8217 & $\mathbf{4 0}$ & 122.5863 & & \\
\hline
\end{tabular}

Therefore, given the temperature interval between $T_{0}=T_{1}=300 \mathrm{~K}$ and $T_{2}=600 \mathrm{~K}$ to the left interface of the lead wall, temperatures at different locations at any time within the lead wall can be now determined.

\section{DIGITAL RESULTS}

The results of the analytical solution of the EDP, $T_{\text {analytical }}(x, t)$, as resolved in section "Theory to find an analytical solution", are presented graphically below. These results are compared with those digital results obtained by simulation in COMSOL Multiphysics, $T_{\text {COMSOL }}(x, t)$. The discrepancy between the analytical solution and COMSOL Multiphysics digital simulation was quantified based on the normalized root-mean-square error (NRMSE) [23], which is defined as:

$$
\operatorname{NRMSE}[\%]=\sqrt{\frac{1}{m} \frac{\sum_{i=1}^{m}\left(T_{i, \text { analytical }}-T_{i, \text { COMSOL }}\right)^{2}}{\sum_{i=1}^{m}\left(T_{i, \text { COMSOL }}\right)^{2}}} \times 100
$$

where $m$ is the number of the data points for one graph, $T_{i \text {,analytical }}$ and $T_{i, C O M S O L}$ are the analytical solutions and COMSOL Multiphysics solution values, respectively, corresponding to the $i$-th data point $(i=1 \ldots m)$. 
Figure 3. The value of NRMSE (Eq. 43) vs. number $n_{u}$ of members of the sum

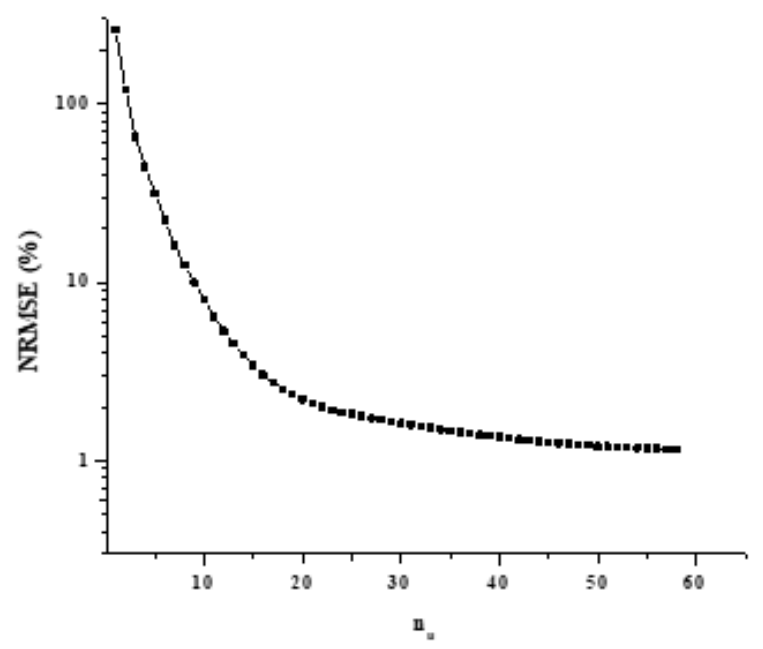

A study of the behavior of the $S S E$ regarding the number of members used in the partial sum of Eq. 41 is presented in Fig. 3. The transient contribution of the analytical solution (Eq. 33) is given as the sum of infinite numbers of the members. To do quantitative calculations, it is necessary to sum a finite number nu of members (Eq. 41), and in this case, calculations were done with numbers of the members from $n_{u}=1$ to $n_{u}=58$, which are shown in Table 1. From Fig. 3 it can be observed that NRMSE decreased as the number of the members was increased, and this became practically stabilized for $n_{u}=$ 57 and 58 values where the difference of the value of NRMSE was smaller than $0.005 \%$.

Figure 4. Spatio-temporal behavior of $\mathrm{T}(\mathrm{x}, \mathrm{t})$ by two different methods: a) The analytical solution $\mathrm{T} \_$analytical $(\mathrm{x}, \mathrm{t})$ for $\mathrm{n} \_\mathrm{u}=58$, and $\mathrm{b})$ The COMSOL Multiphysics digital simulation T_COMSOL $(\mathrm{x}, \mathrm{t})$.
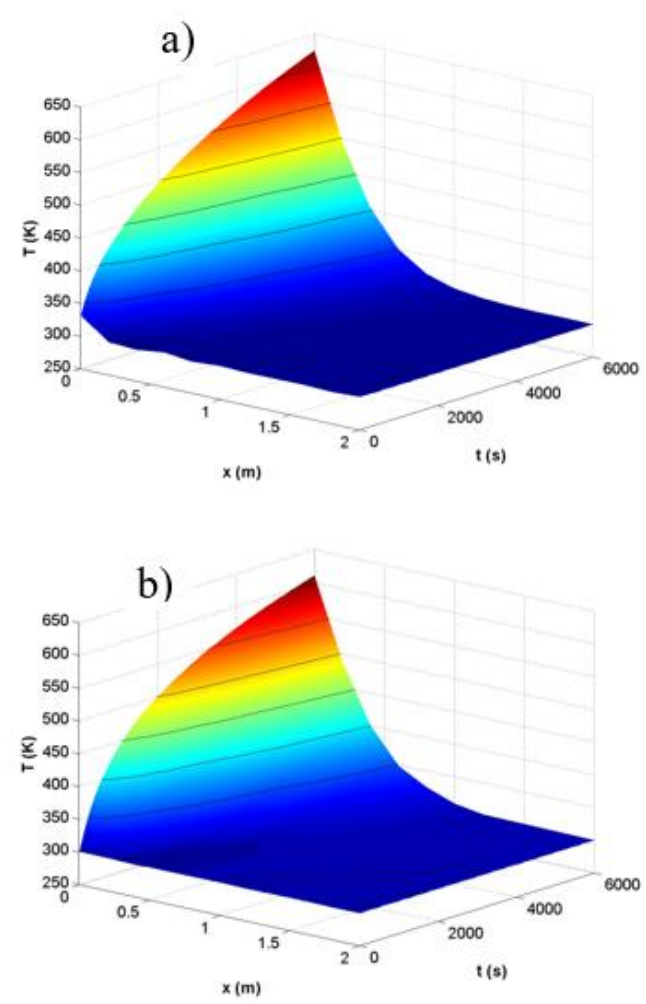
Figure 4-a shows the response surface plot of the temperature as a function of thickness and time generated with the analytical solution, $T_{\text {analytical }}(x, t)$, with $n_{u}=58$ members of the sum (Eq. 41). For comparison, the corresponding response surface plot of the temperature distribution generated with COMSOL Multiphysics digital simulation, $T_{\text {COMSOL }}(x, t)$, is presented in Fig. 4-b:

It was well established that temperature gradients are significant in the layer $2 \mathrm{~m}$ in thickness close to the surface undergoing illumination. That means that reasonable uniform temperature distribution with values similar to $T_{0}$ was observed in the domain $2 \leq x \leq 10 \mathrm{~m}$. Therefore, it was decided to perform the calculations for NRMSE (Eq. 43) and to construct the previous figures only in an interval of $x$ between $0<x<2 \mathrm{~m}$.

\section{EVALUATION OF ERRORS OF THE ANALYTICAL SIMULATION}

Suppose the calculations are made using the analog model, with the set of main thermal parameters of the virtual experiment $\left(\rho(T)\right.$ - the mass density of the material $\left[\mathrm{kg} / \mathrm{m}^{3}\right], c_{p}(T)$ - the specific heat capacity $[\mathrm{J} /(\mathrm{kg} \cdot \mathrm{K})], k(T)$ - the thermal conductivity $[\mathrm{W} /(\mathrm{m} \cdot \mathrm{K})], h(T)$ - the heat loss coefficient $\left.\left[\mathrm{W}\left(\mathrm{m}^{2} \cdot \mathrm{K}\right)\right]\right)$ with its values for extreme temperatures of $T_{1}=300 \mathrm{~K}$ and $T_{2}=600 \mathrm{~K}$, respectively, $T_{0}=$

$300 \mathrm{~K}$. Let their corresponding solutions be $T_{1}^{\text {analogic }}(x, t)$ and $T_{2}^{\text {analogic }}(x, t)$, respectively. The first part of the hypothesis is that the analogic solution of the PDEs of heat diffusion using the statistical values of the main thermal parameters (Eq. 10 to 13), written down as $T_{\text {stat }}^{\text {analogic }}(x, t)$, for all the values of $x$ and of $t$, satisfies the inequality:

$$
T_{1}^{\text {analogic }}(x, t)>T_{\text {stat }}^{\text {analogic }}(x, t)>T_{2}^{\text {analogic }}(x, t)
$$

The simulation in COMSOL Multiphysics is done using the thermal properties values corresponding to the same temperatures, will give results $T_{1}^{\operatorname{COMSOL}}(x, t)$ and $T_{2}^{C O M S O L}(x, t)$, respectively. The second part of the hypothesis is that the solution of the simulation with COMSOL Multiphysics of the PDEs of heat diffusion, written down as $T^{\operatorname{COMSOL}}(x, t)$, for all the values of $x$ and of $t$, satisfies the inequality:

$$
T_{1}^{\operatorname{COMSOL}}(x, t)>T^{\operatorname{COMSOL}}(x, t)>T_{2}^{\operatorname{COMSOL}}(x, t)
$$

The calculations demonstrate that the hypothesis is accomplished in both parts. To be able to present the results $T_{1}^{\text {analogic }}(x, t), T_{\text {stat }}^{\text {analogic }}(x, t)$ and $T_{2}^{\text {analogic }}(x, t)$ of the analytical solution in a 2D graph and at the same time not to lose the generality, the temperature is averaged by $x$, which is: 


$$
T_{\text {averaged by } x}(t)=\frac{1}{2} \int_{x=0}^{x=2} T(x, t) d x
$$

In such a case, the following graph (Fig. 5-a) is available for the analytical solution, where "Aver300A" is the temperature averaged by $x$ for the analytical solution with thermal parameters for 300 $\mathrm{K}($ Aver = averaged). "AverAn" is the temperature averaged by $x$ for the analytical solution with thermal statistical parameters. "Aver600A" is the temperature averaged by $x$ for the analytical solution with thermal parameters for $600 \mathrm{~K}$. As expected, the temperatures for the thermal properties for $300 \mathrm{~K}$ are higher. The temperatures for the thermal properties for $600 \mathrm{~K}$ are lower than the three curves at all times, which is, inequality is accomplished (Eq. 44).

Figure 5. The temperatures averaged by x calculated by a) analytical solution and b) COMSOL Multiphysics simulation. Curves with square points corresponding to T_1, triangle points corresponding to T_2, and circle points corresponding to a) statistical values (Eq. 10 to 13) and b) non-linear solution with COMSOL.
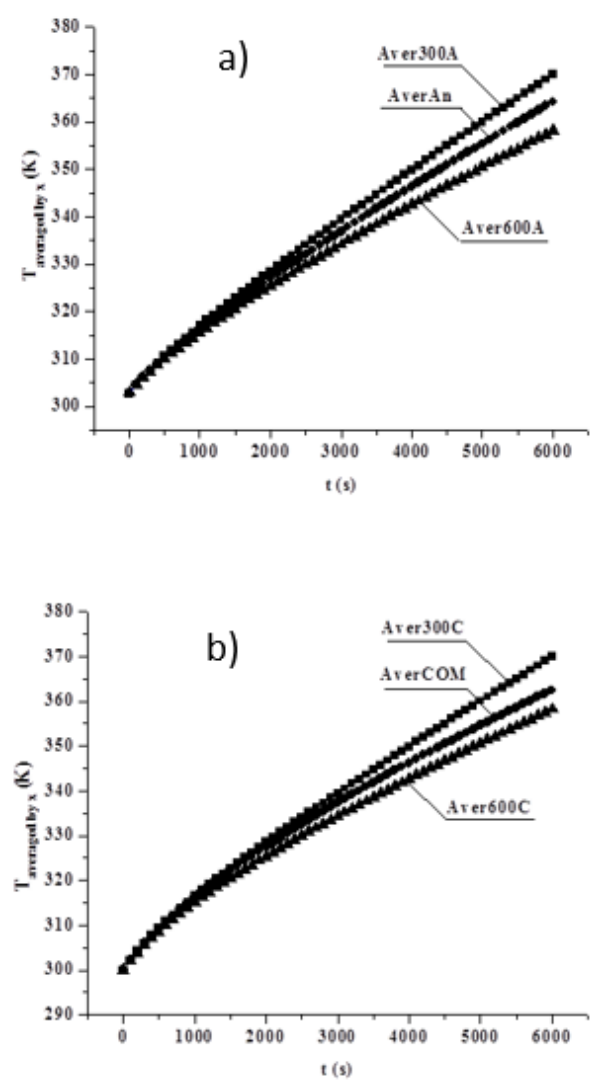

For the simulation with COMSOL Multiphysics, results are shown in Fig. 5-b, where "Aver300C" is the temperature averaged by $x$ for the simulation COMSOL Multiphysics with thermal parameters for $300 \mathrm{~K}$. "AverCOM" is the temperature averaged by $x$ for the simulation COMSOL Multiphysics. "Aver600C" is the temperature averaged by $x$ for the simulation COMSOL Multiphysics with thermal parameters for $600 \mathrm{~K}$. It can be seen that inequality is accomplished (Eq. 45). 
Placing the two graphs of Fig. 5-a, and Fig. 5-b, in a coordinates system, we see that there is a satisfactory coincidence (Fig. 6):

Figure 6. Comparison of the temperatures averaged by $\mathrm{x}$ of the analytical solution and the simulation with the program COMSOL Multiphysics of the Fig. 5-a, and Fig. 5-b. The averaged temperatures are shown as follows: "Aver300A" - black squares; "AverAn" - red circles; "Aver600A" - blue triangles; "Aver300C" - green triangles; "AverCOM" - pink triangles; "Aver600C" - brown triangles

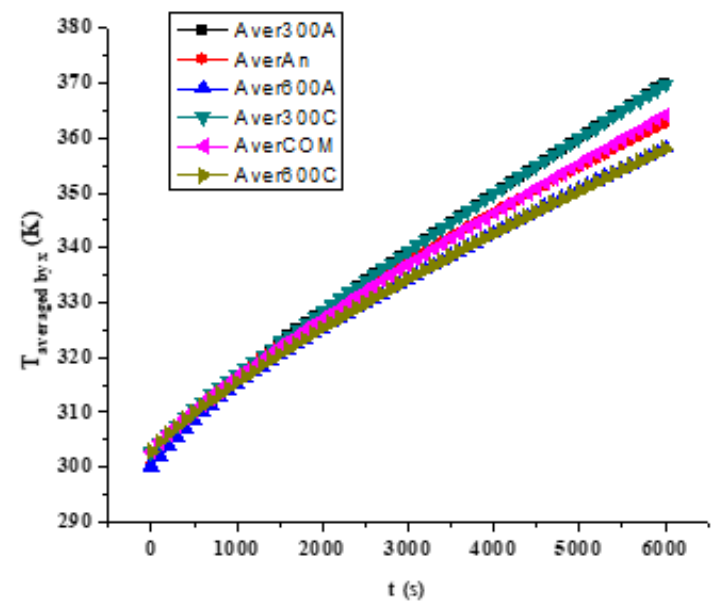

The maximum relative discrepancy for the three pairs of the curves is smaller than $0.1 \%$. That allows the interval $\left[T_{600 K}(x, t) ; T_{300 K}(x, t)\right]$ to be used as representative of the analytical solution error. Comparing the relative difference $D R$ between the two surfaces $(0<x<2 \mathrm{~m}, 0<t<6000 \mathrm{~s})$ : first $T_{1}^{\text {analogic }}(x, t)$ and $T_{2}^{\text {analogic }}(x, t)$ and then $T_{1}^{C O M S O L}(x, t)$ and $T_{2}^{C O M S O L}(x, t)$. The calculation of $D R$ is done by the following equations:

$$
D R_{\text {analytical }}[\%]=\sqrt{\frac{1}{m} \sum_{i=1}^{m}\left(\frac{T_{2}^{\text {analogic }}(x, t)-T_{1}^{\text {analogic }}(x, t)}{T_{2}^{\text {analogic }}(x, t)+T_{1}^{\text {analogic }}(x, t)}\right)^{2}} \times 100
$$

and

$$
\operatorname{DR}_{\text {COMSOL }}[\%]=\sqrt{\frac{1}{m} \sum_{i=1}^{m}\left(\frac{T_{2}^{C O M S O L}(x, t)-T_{1}^{\text {COMSOL }}(x, t)}{T_{2}^{\text {COMSOL }}(x, t)+T_{1}^{\text {COMSOL }}(x, t)}\right)^{2}} \times 100
$$

where $m$ is the number of the data points for one graph or surface, corresponding to the $i$-th data point $(i=1 \ldots m)$. For the indicated surfaces the relative differences $D R$ show the values: $D R_{\text {analytical }}=$ $2.26 \%$ and $D R_{C O M S O L}=2.23 \%$, equal considering. 
Figure 7. The relative difference DR_analytical calculated according to Eq. 47 (red dots) and the relative difference DR_COMSOL of the Eq. 48 for the data of the simulation with COMSOL Multiphysics (black dots).

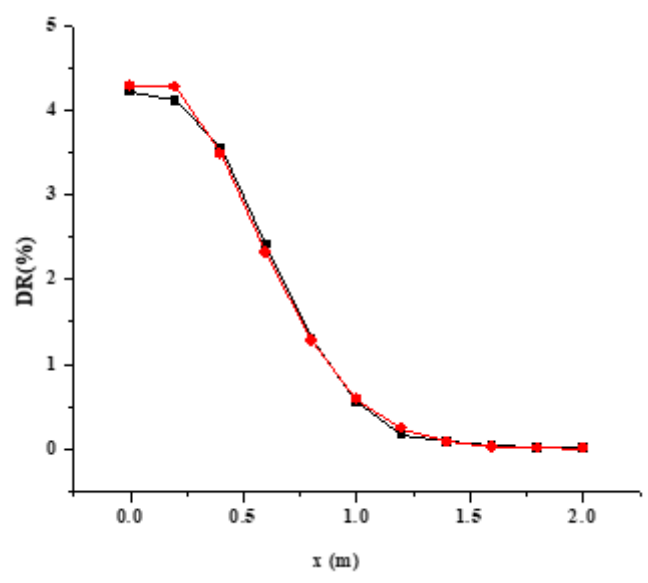

To see if there is a more in-depth match between $D R_{\text {analytical }}$ and $D R_{C O M S O L}$, the values of these two parameters for a row of the matrix $T(x, t)$ are calculated with the scan time $t(0<t<6000 \mathrm{~s})$ and with the family parameter of $x$ curve values. The results are presented in Fig. 7.

An excellent concordance between both curves is seen. That allows the possibility of exchanging the data for the relative difference between both cases - COMSOL Multiphysics and the analytical solution, and it explains the practical equality between $D R_{\text {analytical }}$ and $D R_{\text {COMSOL }}$ for the surfaces $T(x, t)$.

Figure 8. NRMSE vs. $\mathrm{x}$ for scanning time $\mathrm{t}$ from 0 until $6000 \mathrm{~s}$.

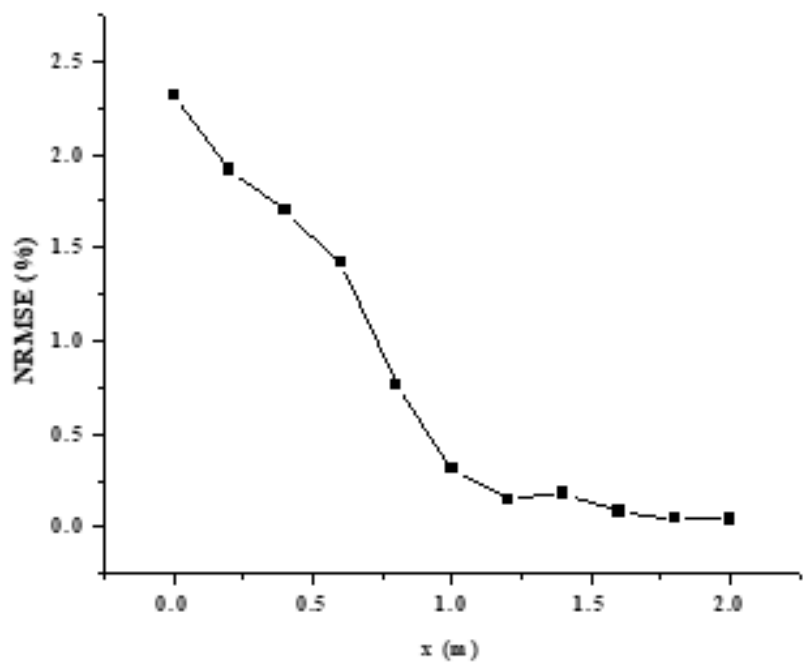

The value of NRMSE among the data of the simulation of COMSOL Multiphysics - our "golden solution," and the results of the analytical solution for all the surface $T(x, t)$ is (Eq. 43$)$ : $N R M S E=1.16$ $\%$. 
Figure 8 shows the calculated values of $N R M S E$ for each row of the matrix $T(x, t)$ making a scan at time $t(0<t<6000 \mathrm{~s})$ and with the family parameter of $x$ curve values.

Figure 9 shows a comparison of the curves of the previous parameters with the standardized $N R M S E$. Standardization is done with respect to the quotient:

$$
C=D R_{\text {analytical }} / N R M S E=2.26 \% / 1.16 \%=1.9482 \approx 2
$$

The similarity between the curve of $D R_{\text {analytical }}$ and the standardized NRMSE curve is noticeable; the relative error between these two curves reaches the value (using the Eq. 43): $N R M S E$ (between the two curves) $=3.40 \%$, that is a relatively small error.

Subsequently, it can be presented the value of NRMSE between COMSOL Multiphysics and analytical solution: $N R M S E \approx D R_{\text {analytical }} / 2$. The value of $D R_{\text {analytical }}$ can be found without the necessity to use COMSOL Multiphysics.

Therefore it is recommended the following work procedure:

1. The wall material and temperatures of interest are chosen: environment temperature $T_{0}$, minimum temperature $T_{1}$ and maximum temperature $T_{2}$.

2. The values of the main thermal properties are looked for: $\rho(T)$ - the mass density of the material $\left[\mathrm{kg} / \mathrm{m}^{3}\right], c_{p}(T)$ - the specific heat capacity $[\mathrm{J} /(\mathrm{kg} \cdot \mathrm{K})], k(T)$ - the thermal conductivity $[\mathrm{W} /(\mathrm{m} \cdot \mathrm{K})]$ as a function of temperature $T$ within the interval $\left[T_{1}, T_{2}\right]$. That could be done based on the corresponding charts in the literature.

Figure 9. The DR_analytical (red dots), DR_COMSOL (black dots), NRMSE (blue dots) and NRMSE standardized by the coefficient C (Eq. 49, green dots) vs. x.

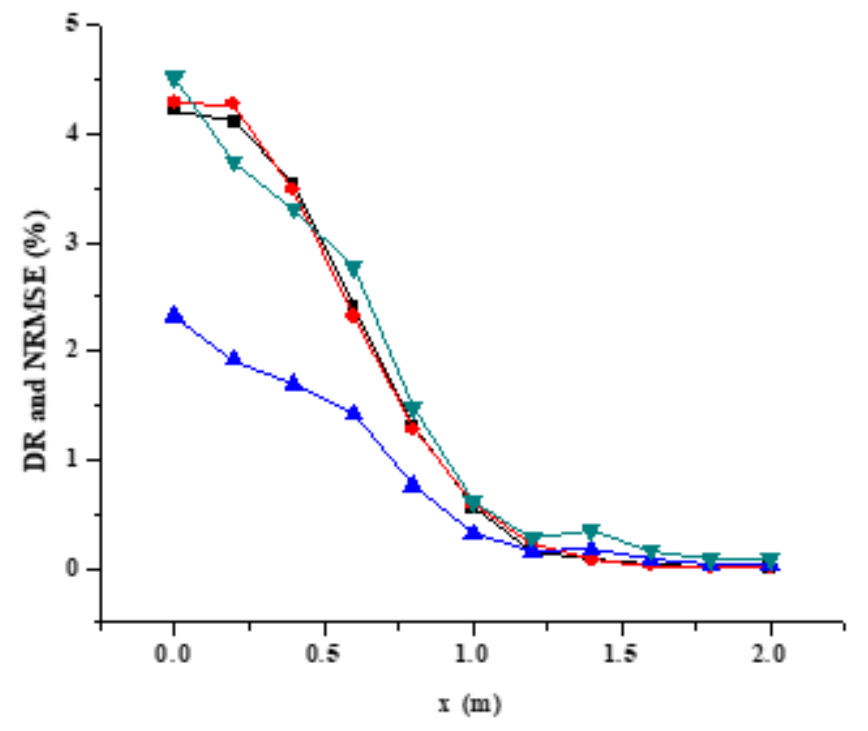


3. Calculations are done for $\rho^{\text {stat }}, c_{p}^{\text {stat }}, k^{\text {stat }}, h^{\text {stat }}$.

4. Heat diffusion equations are analytically resolved with these parameters. The solution is obtained as $T_{\text {stat }}^{\text {analogic }}(x, t)$ within the interval $\left[T_{1}, T_{2}\right]$.

5. With the values of these thermal properties: $\rho, c_{p}, k, h$, for the temperatures $T_{1}$ and $T_{2}$, respectively, analytical solutions are looked for: $T_{1}^{\text {analogic }}(x, t)$ and $T_{2}^{\text {analogic }}(x, t)$, respectively. Using the Eq. 47 the $D R_{\text {analytical }}$ discrepancy between the two solutions is sought. In such case the approximate value of $N R M S E$ between the result of the digital simulation with COMSOL Multiphysics (our "golden method") $T^{\operatorname{COMSOL}}(x, t)$ and the analytical solution $T_{\text {stat }}^{\text {analogic }}(x, t)$, is obtained dividing $D R_{\text {analytical }}$ by 2 .

Of course, this procedure, with other materials and other configurations, must be verified.

\section{DISCUSSION}

A method to linearize heat diffusion PDEs with their boundary and initial conditions in a wide interval of temperatures is presented. One of the advantages of this linearization method is that it is possible, in many experimental configurations, to look for the analytical solution of the problem. Even if for other experimental configurations, this is not possible, for the digital solution of linearized heat diffusion PDEs simple programs can be used to resolve linear PDEs, which are part of traditional software: Mathematica [24], PTC Mathcad [25], EXCEL [26], etc. Another advantage is that the relative error between the analytical solution and the digital simulation with COMSOL Multiphysics can be found without the necessity to own and use the program COMSOL Multiphysics. It is noticed that a big part of the discrepancy between the analytical solution and simulation COMSOL Multiphysics is concentrated closer to the initial moment $t=0$.

\section{ACKNOWLEDGMENT}

The authors thank their respective affiliation institutions (Autonomous University of San Luis Potosi and Autonomous University of Zacatecas) for the support received. 


\section{REFERENCES}

1. Marín, E., Lara-Bernal, A., Calderón, A., and Delgado-Vasallo, O., 2011, "On the heat transfer through a solid slab heated uniformly and continuously on one of its surfaces," Eur. J. Phys., 32(3), pp. 783-791. DOI: 10.1088/0143-0807/32/3/014

2. Peng, X.F., and Peterson G.P., 1996, "Convective heat transfer and flow friction for water flow in microchannel structures," International Journal of Heat and Mass Transfer, 39(12), pp. 2599-2608. DOI: 10.1016/0017-9310(95)00327-4

3. Khan, T.S., Khan, M.S., Chyu, Ming-C., and Ayub, Z.H., 2010, "Experimental investigation of single phase convective heat transfer coefficient in a corrugated plate heat exchanger for multiple plate configurations," Applied Thermal Engineering, 30(8-9), pp. 1058-1065. DOI: 10.1016/j.applthermaleng.2010.01.021

4. Ayub, Z.H., 2003, "Plate Heat Exchanger Literature Survey and New Heat Transfer and Pressure Drop Correlations for Refrigerant Evaporators," Heat Transfer Engineering, 24(5), pp. 3-16. DOI: 10.1080/01457630304056

5. Palyvos, J.A., 2008, "A survey of wind convection coefficient correlations for building envelope energy systems' modeling," Applied Thermal Engineering, 28(8-9), pp. 801-808. DOI: 10.1016/j.applthermaleng.2007.12.005

6. Churchill, S.W., and Chu, H.H.S., 1975, "Correlating equations for laminar and turbulent free convection from a vertical plate," International Journal of Heat and Mass Transfer, 18(11), pp. 1323-1329. DOI: 10.1016/0017-9310(75)90243-4

7. Lienhard IV, J.H. and Lienhard V, J.H., 2019, A Heat Transfer Textbook, 5th Ed., Dover Publications, Mineola, NY. ISBN: 9780486837352

8. Jones, B.J., McHale, J.P., and Garimella, S.V., 2009, "The Influence of Surface Roughness on Nucleate Pool Boiling Heat Transfer," J. Heat Transfer, 131(12), pp. 121009-121023. DOI: $10.1115 / 1.3220144$

9. Lewis, M.J., 1974, "Roughness Functions, the Thermohydraulic Performance of Rough Surfaces and the Hall Transformation - an Overview," Int. J. Heat Mass Transfer, 17(8), pp. 809-814. DOI: 10.1016/0017-9310(74)90149-5

10. Ay, H., Jang, J. Y., and Yeh, J.N., 2002, "Local heat transfer measurements of plate finned-tube heat exchangers by infrared thermography," International Journal of Heat and Mass Transfer, 45(20), pp. 4069-4078. DOI: 10.1016/S0017-9310(02)00132-1

11. Miettinen, L., Kekäläinen, P., Merikoski, J., and Timonen, J., 2009, "Measurement of the In-plane Thermal Diffusivity and Temperature-Dependent Convection Coefficient Using a Transient Fin Model and Infrared Thermography," Int J Thermophys, 30(1902), pp. 1902-1917. DOI: 10.1007/s10765-0090690-3

12. Landolt-Bornstein, 2005, "Numerical Data and Functional Relationships in Science and Technology: New Series III," edited by Madelung, O. and White, G. K., 2.3.1.11. Thermal Transport pp. 2-217 (Springer: Berlin, 2005). ISBN: 3-540-16609-2 
13. Salazar, A., Mendioroz, A., and Fuente, R., 2009, "The strong influence of heat losses on the accurate measurement of thermal diffusivity using lock-in thermography," Applied Physics Letters, 95(12), pp. 121905-1-3. DOI: 10.1063/1.3236782

14. Mendioroz, A., Fuente-Dacal, R., Apiñaniz, E., and Salazar, A., 2009, "Thermal diffusivity measurements of thin plates and filaments using lock-in thermography," Review of Scientific Instruments, 80(7), pp. 074904-1-9. DOI: 10.1063/1.3176467

15. Xu, J., and Fisher, T.S., 2006, "Enhancement of thermal interface materials with carbon nanotube arrays," International Journal of Heat and Mass Transfer, 49(9-10), pp. 1658-1666. DOI:10.1016/j.ijheatmasstransfer.2005.09.039

16. Ristau, D., and Ebert, J., 1986, "Development of a thermographic laser calorimeter," Applied Optics, 25(24), pp. 4571-4578. DOI: 10.1364/ao.25.004571

17. Marín, E., 2009, “Linear relationships in heat transfer,” Lat. Am. J. Phys. Educ., 3(2), pp. 243 245.

18. Martínez, K., Marín, E., Glorieux, C., Lara-Bernal, A., Calderón, A., Peña Rodríguez, G., and Ivanov, R., 2015, "Thermal diffusivity measurements in solids by photothermal infrared radiometry: Influence of convection-radiation heat losses, " International Journal of Thermal Sciences, 98(12), pp. 202207. DOI: 10.1016/j.ijthermalsci.2015.07.019

19. Salazar, A., Apiñaniz, E., Mendioroz, A., and Oleaga, A., 2010, "A thermal paradox: which gets warmer?,” Eur. J. Phys., 31(5), pp. 1053-1059. DOI: 10.1088/0143-0807/31/5/007

20. COMSOL Multiphysics®. www.comsol.com. COMSOL AB, Stockholm, Sweden.

21. Comex: https://www.comex.com.mx/

22. Usamentiaga, R., Venegas, P., Guerendiaga, J., Vega, L., Molleda, J., and Bulnes, F.G., 2014, "Infrared Thermography for Temperature Measurement and Non-Destructive Testing," Sensors, 14(7), pp. 12305-12348. DOI: $10.3390 / \mathrm{s} 140712305$

23. Ivanov, R., Marín, E., Cruz-Orea, A., Pichardo-Molina, J.L., Moreno, I., and Araujo-Andrade, C., 2009, "Differential sensor in front photopyroelectric technique: II. Experimental," J. Phys. D: Appl. Phys., 42(12), pp. 125504-1-8. DOI: 10.1088/0022-3727/42/12/125504

24. Wolfram ${ }^{\circledR}$, https://www.wolfram.com/.

25. Mathcad®, https://www.ptc.com/es/products/mathcad.

26. ExcelWorks LLC®, PDSOLVE, https://excel-works.com/manual/pdsolve. 